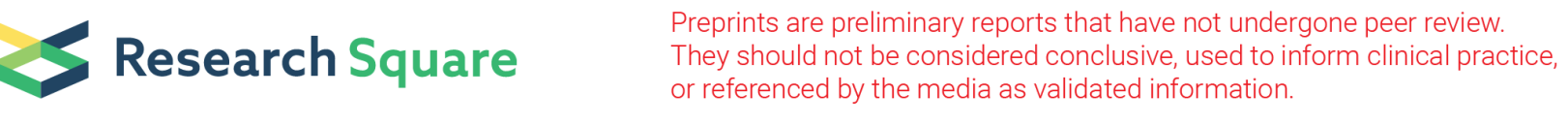

\title{
Five-year survival in patients with Glioblastoma is overestimated in registry data - A nationwide population- based Swedish survey during 1958 - 1999
}

Peter Milos ( $\sim$ peter.milos@regionostergotland.se )

Linköping University

\section{Edward Visse}

Lund University

Abdulrahman Al-Shudifat

The University of Jordan

Peter Siesjö

Lund University

\section{Research Article}

Keywords: Glioblastoma, five-year survival, cohort, Sweden, pre temozolomide.

Posted Date: June 4th, 2021

DOI: https://doi.org/10.21203/rs.3.rs-561751/v1

License: (c) (i) This work is licensed under a Creative Commons Attribution 4.0 International License. Read Full License 


\section{Abstract}

Background Some glioblastoma (GBM) patients survive more than five years with hitherto no clearly established epidemiological or molecular causes. Since varying rates of GBM five-year survival have been reported our aim was to assess the true prevalence of five-year survivors in Sweden from 1958 to 1999, before the introduction of concomitant temozolomide treatment.

Methods After screening the Swedish Cancer Registry and the Cause of Death Register 736 out of 12765 patients with highgrade glioma were defined as five-year survivors. The full text pathology report was reviewed in 585 patients. Data on epidemiology and treatment were retrieved from the medical records of 556 patients.

Results 77 five-year survivors with primary GBM were identified which corresponded to $0.60 \%$ of the initial population. During 1990 to 1999 GBM five-year survival was 0. 90\%. Younger age, Karnofsky score $>70$ and non-eloquent tumour location were found in most but not all five-year survivors.

Conclusion GBM five-year survival was exceedingly rare in Sweden until 2004, comprising less than $1 \%$ of registered HGGs. Relying on registry data without reviewing the pathology report will overestimate the accurate number of five-year survivors. To our knowledge, this is the only nationwide population-based study of five-year survival in GBM patients.

\section{Introduction}

Glioblastoma (GBM), the most common malignant brain tumour, carries the worst prognosis of all human tumours, with even shorter survival than in pancreatic cancer and mesothelioma [1]. GBM usually grows aggressively with excessive seeding of tumour cells outside the bulk of the tumour, often infiltrating vital functional brain areas making radical surgical resection impossible [2-4]. The biology of GBM is overly complex with genetic and molecular heterogeneity both between patients but also within the same patient making targeted oncological therapy challenging [5-7]. The current standard treatment for newly diagnosed GBM patients is maximal safe surgery followed by radiotherapy with concomitant temozolomide and then adjuvant temozolomide [8]. The median survival in patients receiving this regimen is about 14 months [9]. However, due to that not all patients receive standard therapy, median survival for all diagnosed GBM patients is considerably less, being reported from 5.8 to 11.2 months [10-13]. Certain clinical and molecular characteristics such as younger age, good performance status and gross total surgical resection have been associated with longer survival together with 06-methylguanine methyltransferase (MGMT) promoter methylation status [14-21]. Recently, studies have indicated a remarkable gain in overall survival in subgroups of GBM patients [22-24], but even in the most favourable conditions the majority succumb to the disease within 2 years [25]. However, a subset of GBM patients seem more amenable to treatment, having longer progression free and overall survival and are called Long Term Survivors (LTS). The definition of LTS varies but is commonly defined as survival $>36$ months [26]. An even smaller minority survives five years or more after diagnosis. In the original study by Stupp et al five-year survival increased from 2 to $10 \%$ when concomitant temozolomide treatment was introduced and compared with radiotherapy alone [37]. However, numerous reports on five-year survival both before and after the introduction of concomitant therapy differ considerably between 1-10 \% depending on population sample, selection method, study design, accuracy of diagnosis and performed interventions [1,10-13,24-36]. Clinical trials and single institutional studies tend to have higher numbers of five-year survivors [21, 25, 37] than population-based studies [10-13]. There is also a concern about the true number of five-year survivors due to misdiagnosis and the varying definition of anaplastic astrocytoma, oligodendroglioma and oligoastrocytoma depending on time and place [28, 38, 39]. Although the reported numbers need to be interpreted cautiously, some GBM patients have undoubtedly benefitted from the last decade's treatment algorithms with a prolonged overall survival, especially concerning two and three-year survival but not necessarily five-year survival [75]. In the post temozolomide era, studies investigating molecular factors in GBM LTS patients has been somewhat conflicting indicating MGMT promoter methylation as a strong predictor for long term survival whereas the role of isocitrate dehydrogenase (IDH) mutations are less clear [15,40-42]. Previous case reports have implied an association between postoperative infections and LTS [43] but recent retrospective reports have not been able to confirm this [44, 45]. 
Autoimmunity has been reported to prolong survival in glioma [46] but this has not been verified in other series [47]. Nevertheless, GBM patients with extended survival may display important prognostic factors for longer survival, hopefully enabling new targets for treatments in the future.

Historically there has been a gradual implementation of new surgical techniques and oncological treatments for GBM since 1960 but the impact of these methods has not been estimated in population-based studies. It is important to assess these changes to define the survival status before the introduction of temozolomide in the concomitant and adjuvant settings. The aim of this study was therefore to identify GBM five-year survivors in Sweden during 1958 - 1999, before the introduction of temozolomide into clinical practice around 2004, gathering epidemiologic data and searching for clinical features predisposing for five-year survival.

\section{Materials And Methods}

After ethical approval, a request was made to the Swedish National Board of Health and Welfare to extract data of HGG patients from the Swedish Cancer Registry and the Swedish Cause of Death Register from 1958 to 1999 with the topography codes 193.0, 191-9 and C71.0-9 (according to ICD 7, ICD 9 and ICD 10) and morphology codes 476 (before ICD-0); 93803, $94403,94013,94413,94423$ (from ICD-0 after 1990). Social security numbers of HGG patients (= all ages) with at least fiveyear survival were then gathered and extracted.

The Swedish Cancer Registry was founded in 1958 and comprises all cancer diagnosis in Sweden. The registry has been reviewed previously with an overall completeness of approximately $98 \%$ [48] and $96 \%$ [49]. However, in the later survey there was a tendency of underreporting tumours of the nervous system, especially in elderly patients over 70 years. In a recent study reviewing malignant brain tumours in the registry between $1990-2014$, the completeness was approximately $90 \%$ in the younger population (< 70 years) but considerably less in elderly patients (> 70 years), ranging between $65-75 \%$ [50].

\section{Patients}

12765 patients with a diagnosis of HGG were identified. 736 of these were defined as five-year survivors. The aim was to review the full pathology report, neurosurgical and oncological charts in these patients from the six neurooncological regions in Sweden (South, Southeast, Stockholm, West, Middle and North). 585 full pathology reports and 556 medical charts were found. 151 pathology reports and 180 charts were irretrievable (fig 1). The lost charts were mainly from the earlier decades (1958-1969; 1970-1979 - 145 patients, 80\%). However, all full pathology reports from patients in the last decade (1990-1999) were retrieved. Data regarding previous disease, presenting symptoms, functional status, previous history of trauma, infection and tumor, radiological exams, neurosurgical operations, re-operations, diagnosis, oncological treatment, and overall survival was extracted from 556 patients with complete medical charts.

Surgical resection was scored as gross total resection (GTR), partial resection or biopsy. Since postoperative MRI was not performed routinely in Sweden until the beginning of the 21 st century, resection grade was estimated after reviewing the neurosurgical operative report, i.e. the surgeon's report. Epidemiological data was collected into a database (File Maker Pro and Excel) for graphics and further statistical analysis.

\section{Statistical methods}

Statistical analyses were performed with the free statistical software R-project

(R core team (2017). R: A language and environment for statistical computing. R Foundation for Statistical Computing, https://www.R-project.org/) 
Standard descriptive statistics, tables and diagrams were used to display trends and epidemiological data. Differences in overall survival between different age groups and time periods were compared with the Kaplan-Meier log rank test.

\section{Ethical approval}

Ethical approval was obtained from the Regional Ethical Review board in Lund, Sweden (Dnr 617/2004). All research was performed in accordance with the Declaration of Helsinki. Informed consent was obtained from alive adult patients and legal guardians from patients $<18$ years.

\section{Results}

\section{Survival in patients with high-grade glioma was related to time period and age}

In the population of 12765 HGG patients, demographics showed a peak in incidence in the sixth and seventh decade (fig 2). There was a highly significant difference $(p<0.001)$ in median survival between the three first time cohorts $(1958-1969$ [2.3 months; 80 days]; 1970-1979 [4.8 months; 145 days]; 1980-1989 [7.1 months; 213 days]) and a significant difference ( $p=$ 0.017) between the two last cohorts (1980-1989 [7.1 months; 213 days]; 1990-1999 [8.4 months; 252 days]) (fig 3a). When adjusted for the complete number of GBM five-year survivors in the 1990 - 1999 time cohort the median survival was reduced to 7.8 months; 233 days (fig. 3b). There was a tendency to an augmented number of HGG five-year survivors in the latter cohorts (table 1). After stratifying the population into different age cohorts (0-9; 10-19; 20-29; 30-39; 40-49; 50-59; 6069; 70-79; $80-w$ years) there was a stepwise significant difference between all age groups over 39 years (median survival 5.6 months ; 168 days for the whole population and for the different age groups: $16.2 / 485 ; 20.5 / 615 ; 23.2 / 696 ; 17.2 / 517$; $10.2 / 305 ; 6.9 / 206 ; 4.4 / 132 ; 2.4 / 73 ; 1.1 / 32$ months/days respectively, $\mathrm{p}<0.001$ ) and only a partially significant difference in age groups $<39$ years, with the highest median survival in the 20-29 cohort (fig. 4).

\section{Five-year HGG survivors}

$736(5,8 \%)$ patients with registered HGG had a recorded five-year survival or more. The full pathology reports were reviewed in 585 cases and interpreted according to the 1993 WHO classification of Tumors of the Central Nervous System [48]. The most common diagnosis was Anaplastic Astrocytoma (197 patients), followed by Astrocytoma grade II (86 patients), GBM (77 patients), Oligoastrocytoma grade III (34 patients), Pilocytic Astrocytoma grade I (27 patients), Oligodendroglioma grade II (26 patients) and Anaplastic oligodendroglioma grade III (24 patients). In eighteen cases no specific diagnosis was found. Three cases were intramedullary gliomas and nineteen were meningiomas that previously had been registered as HGG (table 2).

\section{Glioblastoma patients}

87 patients with a histopathological diagnosis of GBM (mitosis, hypercellularity, vascular proliferation and necrosis) were initially categorized as primary GBM. Patients with a previous diagnosis of diffuse low-grade glioma (DLGG), e.g. secondary GBMs and those with oligodendroglioma features were excluded. However, the primary GBM diagnosis was changed after additional surgery in 10 patients.

Thus, 77 patients were confirmed as primary GBM five-year survivors, constituting $0,60 \%$ of the total population of 12765 patients and $10,5 \%$ of 736 registered five-year survival HGG patients. 39 patients (51\%) were male, 38 patients (49\%) were female. The age at diagnosis ranged from 8-69 years with a median age of 41 years. Twenty-one patients were diagnosed in the age group 40-49 years, followed by 15 patients in 30-39 group, 14 patients in the 50-59 group, 12 patients in the 20-29 group, 8 patients in the 60-69 group, 6 patients in the 10-19 group, and 1 patient in the 0-9 age group (fig 5 a). 55 patients (71\%) were younger than 50 years and 22 patients (29\%) were older than 50 years when diagnosed.

Time periods and geographical location 
Most GBM five-year survivors, 48 patients (62\%), were diagnosed and treated in in the latter decades (1980-1989; 19901999) whereas 29 patients (38\%) were treated in the earlier decades (1958-1969; 1970-1979) (table 1).

\section{Symptoms and signs}

Medical charts from four patients where the full pathology report had verified GBM diagnosis could not be retrieved. These patients were however included in the survey as five-year survivors. Charts from 73 GBM patients were reviewed. The most common presenting symptom was headache in 46 patients (63\%) followed by focal neurological signs in 38 patients (52 \%), nausea and vomit in 28 patients (38\%), seizure in 26 patients (36\%) and vertigo in 18 patients (25\%). 6 patients (8\%) had a history of autoimmunity (allergy, asthma, and other autoimmune diseases). No patients had multiple sclerosis. 6 patients $(8 \%)$ have had previous head trauma. 3 patients $(4 \%)$ had a heredity of brain tumours and 6 patients (8 \%) of other tumours. 5 patients $(7 \%)$ have had previous non-CNS tumours, and none have had other brain tumours beside glioma.

Six patients (8\%) had been treated for a general bacterial infection prior to surgery. 6 patients (8\%) developed a local, superficial wound infection postoperatively treated with oral antibiotics and 6 patients $(8 \%)$ had a deep postoperative infection treated with surgery, intravenous and later oral antibiotics. Three patients with local wound infection eventually developed and were treated for a deep wound infection. Thus, 9 patients $(12 \%)$ were treated for a deep postoperative wound infection. The median overall survival in patients with a postoperative infection was 2663 days (7,3 years) which was less than the overall survival in the non-infected population (median survival $\approx 9$ years) (table 3 ).

51 patients (70\%) had a preoperative Karnofsky functional status score $>70$ and 22 patients (30 \%) had $<70$ (table 3 ).

\section{Tumour location}

GBM was located in the frontal lobe in 40 patients (55\%), temporal lobe in 14 patients (19\%), parietal lobe in 8 patients (11 $\%)$, occipital lobe in 6 patients (8\%), ventricle in 2 patients (2,8 \%), cerebellum and brain stem in 1 patient (1\%). In one patient $(1 \%)$ the tumour was multi lobular. 44 patients $(60 \%)$ had tumours in the right hemisphere whereas 29 patients (39

$\%)$ had tumours in the left. Forty-three tumours (59\%) were located superficially $(<1 \mathrm{~cm}$ below cortex), 30 tumors $(41 \%)$ were deeply located (> $1 \mathrm{~cm}$ below cortex) (table 3 ).

\section{Treatment}

36 patients (49\%) were operated with Gross Total Resection (GTR) according to the surgeon's report. 32 patients (44\%) had partial resection and 5 patients $(7 \%)$ underwent a biopsy. 24 patients $(33 \%)$ had repeated surgery whereas 49 patients $(67 \%)$ did not have any secondary surgery. Among patients with repeated surgery, 19 patients had one, 4 patients had two and one patient had three additional surgeries. Almost all, 69 patients (95\%) received postoperative oncological treatment with full radiotherapy (56-60 Gy) whereas 4 patients $(5 \%)$ did not. 38 patients (52\%) did also receive chemotherapy (table 3 ).

\section{Sample evaluation}

The full neuropathology reports were reviewed by the authors as described above. Thirty-three patient samples had been reexamined during the clinical course due to an unexpectedly long survival, warranting a new examination. In additionally twenty-four patients, the diagnosis of GBM was confirmed through iterated surgeries. In sixteen patients, no new evaluation had been made. Hence, in 57 out of 73 cases the GBM diagnosis had been reexamined and verified. Additional microscopic sample examinations were not performed by the authors.

\section{Overall Survival}

Overall survival in GBM five-year survivors ranged between 5-55 years with a median survival of 3251 days or approximately 9 years (8 years, 11 months) (fig 5b). Ten patients were still alive in January 2020. Nineteen patients (24\%) survived five but 
less than six years after diagnosis, representing the largest cluster in the cohort. Thirty-five patients $(0.27 \%)$ survived more than ten years.

\section{Discussion}

Most of the registered HGG five-year survivors had an actual diagnosis of anaplastic astrocytoma, astrocytoma grade 2 and other non GBM diagnoses. Of the 585 HGG patients with a verified diagnosis, only 77 had a diagnosis of primary GBM which constituted $0.60 \%$ of the total population, illustrating the potential flaws when extracting data from registries without verifying the diagnosis $[28,39]$. Considering the 151 reports that could not be retrieved the true number of five-year survivors may be slightly higher but supposedly less than $1 \%$ as the $1990-1999$ cohort had a five-year survival of 0,9\% (table 1). This number is below the previously reported figures from before year 2000 of $1-2 \%[28,29]$. Recent population-based studies estimated GBM five-year survival between 2.0-2.4 \% [10, 13]. However, in the American study encompassing over 100000 GBM cases only patient data from accredited cancer centers were included thus possibly overestimating the actual five-year survival [10]. Furthermore, no review of pathology was made adding additional uncertainty of the actual numbers of fiveyear survivors. Given that completeness of registry data is less in older patients for brain tumors our estimate might still be

too high [50]. Notably, thirty-five patients $(0.27 \%)$ lived more than ten years after diagnosis which is in proximity to data from a systematic review on ten-year survivors of $0.71 \%$ [52].

Headache was the most common presenting symptom in 73 GBM five-year survivors, followed by focal neurological signs, nausea and seizure, correlating with data from other GBM studies $[4,53,54]$.

Some studies have reported longer survival in diffuse glioma patients with a history of allergy and autoimmune disease whereas this relationship was unclear in GBM patients [55]. Only six (8\%) patients in our study had a history of allergy, asthma or autoimmune disease, which does not support the theory that previous allergy or autoimmune disease have a protective or treatment promoting effect once GBM has been established. However, our findings do not contradict the notion of an inverse association between glioma and allergic conditions, protecting the host from developing glioma [56-61].

Five patients (7\%) had been treated for prior tumours, but none had a history of other brain tumours which is congruent with previous findings by Zacharia et al stating that approximately $8 \%$ of GBM patients had a prior cancer diagnosis and were more likely to harbor an EGFR or MGMT mutation [62]. The overall survival in this group did not seem to be augmented which corresponds to our results where only a minority of the five-year GBM survivors have had previous tumour disease.

Nine patients had a postoperative wound infection with a median OS of 7.3 years. The effect of postoperative infection on survival has been extensively investigated, not finding any clear evidence of a significant impact on five-year survival, corresponding with our results. [44, 45,63].

Most GBM patients had tumours located superficially in the frontal lobe and in the right hemisphere, had a preoperative Karnofsky functional score $>70$ and were younger than 50 years when diagnosed, corroborating that younger patients with a high functional status and non-eloquent located, superficial tumours not in obvious contact with the subventricular zone tend to have better prognosis and a longer overall survival [64-68].

About half of the 73 patients (49\%) were operated with GTR, the rest with either partial resection (44\%) or biopsy (7\%). Twenty-four (33\%) had repeated surgery. This data from the pre-MRI period needs to be interpreted cautiously, being based on surgeon's report, with a reported low accuracy of approximately 30\% [69]. As the extent of resection (EOR) plays a role in GBM survival [14, 22-24, 70-73] the percentage of GTR among GBM five-year survivors in our study seems surprisingly low. Almost all patients (95\%) were treated with radiotherapy whereas a small majority (52\%) received chemotherapy, mainly BCNU during the latter decades. The current standard therapy with concomitant radiochemotherapy followed by adjuvant temozolomide have had a significant impact on overall survival in clinical trials $[9,25,37]$ and also in population-based reports, especially regarding two- and three-year survival $[1,10-13,29,74,75]$. However, some of these reports are based on 
data from the SEER database, a registry that comprises approximately $35 \%$ of the US population with an uneven geographical distribution (https://seer.cancer.gov/data/). Full concomitant radiochemotherapy is mainly given to younger patients deemed clinically fit and only a minority of elderly patients above 65 years receives this regimen. The overall impact of temozolomide on GBM five-year survival is less clear. In a recent meta-analysis five-year survival was not overly affected before and after 2005 when temozolomide treatment was initiated in clinical practice [75]. Similarly, a newly published population based French study reported a five-year survival of 2, $4 \%$ [13]. Other studies report a wide variety of GBM fiveyear survival, ranging from 1-10 \% [1, 10-12, 27-36]. This variation may be caused by selection bias of treated patients and controls but possibly also due to publication bias, e.g. reporting of lower survival might be less frequent.

There has been an increasing focus on molecular markers in GBM LTS, especially MGMT promoter methylation and IDH mutations. MGMT promotor methylation status is well described as a predictive marker of response to temozolomide in GBM. Several studies have shown a high percentage rate of MGMT promoter methylation in GBM LTS, particularly in patients surviving $>3$ years [26, 76-79] but not necessarily in five-year survivors [21]. Mutation in IDH occurs frequently in low grade and anaplastic astrocytoma and is associated with better prognosis. However, this association seems to be weaker and sometimes lacking in GBM LTS $(40-42,78,79]$. Some, especially younger GBM patients with IDH wt together with ATRX mutations, have been reported to have longer overall survival [80] but this has not been corroborated by others [81]. In summary, no single factor has so far been able to explain five-year survival in GBM patients either before or after the introduction of concomitant temozolomide.

\title{
Limitations
}

This study has several limitations. It is retrospective, comprising more than 40 years of the Swedish Cancer Registry. Different brain tumor classifications, errors in registration and lack of completeness are common pitfalls when extracting data from a registry with an obvious risk of gathering and interpreting incorrect data. It is not known how many of the 12765 HGG patients that were true primary GBM and survived less than five years since it would be unfeasible to review the pathology reports for all patients. However, it is probable that non-GBM diagnosis was much less frequent in patients with a survival less than five years. Furthermore, the diagnosis was based on previous pathology assessment and then interpreted according to the $1993 \mathrm{WHO}$ classification. Errors when interpreting the pathology specimen leading to wrong diagnosis has been reported $[35,36]$. However, fifty-seven of the GBM five-year survivors $(75 \%)$ had been reevaluated and hence reviewed by another neuropathologist verifying the diagnosis. Irretrievable charts from approximately $25 \%$ of the initial study population of 736 patients and the lack of postoperative radiology for definitive estimation of tumour residual are also important limitations.

\section{Conclusions}

The actual GBM five-year survival in Sweden was 0,60\% during 1958-1999 and 0.9\% during 1990-1999, prior to implementation of concomitant temozolomide treatment. These numbers are well below reported five-year survival in clinical trials and most population-based studies. Younger age, good preoperative functional status, tumor location and superficially located tumors appears to be the most important clinical prognostic factors for GBM long-term survival but do not explain all five-year survivors. To our knowledge, this is the only nationwide population-based study of five-year survival in GBM patients.

\section{Declarations}

\author{
Acknowledgements: None
}

Author contributions: 
PM: Conceptualization, data curation, formal analysis, investigation, methodology, project administration, resources, software, validation, writing - original draft and writing - review and editing

EV: data curation, formal analysis, investigation, software, methodology, validation, and writing - review and editing AAS: data curation, validation, writing - review and editing

PS: Conceptualization, formal analysis, funding acquisition, investigation, methodology, project administration, software, supervision, validation, writing - original draft, and writing - review and editing.

\section{References}

1. Ostrom QT, Gittleman H, Truitt G, Boscia A, Kruchko C, Barnholtz-Sloan JS. CBTRUS Statistical Report: Primary Brain and Other Central Nervous System Tumors Diagnosed in the United States in 2011-2015. Neuro Oncol. 2018 Oct 1;20(suppl_4):iv1-iv86

2. Kamp MA, Dibué-Adjei M, Cornelius JF et al. Is it all a matter of size? Impact of maximization of surgical resection in cerebral tumors. Neurosurg Rev. 2019 Dec;42(4):835-842.

3. Pessina F, Navarria P, Cozzi L et al. Maximize surgical resection beyond contrast-enhancing boundaries in newly diagnosed glioblastoma multiforme: is it useful and safe? A single institution retrospective experience. J Neurooncol. 2017 Oct;135(1):129-139.

4. Omuro A, DeAngelis LM. Glioblastoma and other malignant gliomas: a clinical review. JAMA. 2013 Nov 6;310(17):184250.

5. Sottoriva A, Spiteri I, Piccirillo SG et al Intratumor heterogeneity in human glioblastoma reflects cancer evolutionary dynamics. Proc Natl Acad Sci U S A. 2013 Mar 5;110(10):4009-14.

6. Spiteri I, Caravagna G, Cresswell GD et al. Evolutionary dynamics of residual disease in human glioblastoma. Ann Oncol. 2019 Mar 1;30(3):456-463.

7. Bernstock JD, Mooney JH, llyas A et al. Molecular and cellular intratumoral heterogeneity in primary glioblastoma: clinical and translational implications. J Neurosurg. 2019 Aug 23:1-9.

8. Weller M, Van den Bent M, Hopkins K et al. European Association for Neuro-Oncology (EANO) Task Force on Malignant Glioma. EANO guideline for the diagnosis and treatment of anaplastic astrocytomas and glioblastoma. Lancet Oncol. 2014 Aug;15(9):e395-403

9. Stupp R, Mason WP, van den Bent MJ, Weller M et al. European Organisation for Research and Treatment of Cancer Brain Tumor and Radiotherapy Groups; National Cancer Institute of Canada Clinical Trials Group. Radiotherapy plus concomitant and adjuvant temozolomide for glioblastoma. N Engl J Med. 2005 Mar 10;352(10):987-96.

10. Dressler EV, Liu M, Garcia CR et al. Patterns and disparaties of care in glioblastoma. Neuro-Oncol Practice 2019; 6:37-49

11. Dubrow R, Darefsky AS, Jacobs DI et al. Time trends in glioblastoma multiforme survival: the role of temozolomide. Neuro Oncol. 2013, Dec;15(12):1750-61.

12. Eriksson M, Hahari J, Vestman A et al. Improved treatment of glioblastoma - changes in survival over two decades at a single regional Centre. Acta Oncol. 2019; 58 (3): 334-341

13. Fabbro-Peray P, Zouaoui S, Darlix A et al. Association of patterns of care, prognostic factors, and use of radiotherapytemozolomide therapy with survival in patients with newly diagnosed glioblastoma: a French national population-based study. J Neurooncol. 2019; 142(1): 91-101

14. Brown TJ, Brennan MC, Li M. et al. Association of the Extent of Resection with Survival in Glioblastoma: A Systematic Review and Meta-analysis. JAMA Oncol. 2016 Nov 1;2(11):1460-1469.

15. Hegi ME, Diserens AC, Gorlia T et al. MGMT gene silencing and benefit from temozolomide in glioblastoma. $N$ Engl J Med. 2005 Mar 10;352(10):997-1003. 
16. Filippini G, Falcone C, Boiardi A et al. Brain Cancer Register of the Fondazione IRCCS (Istituto Ricovero e Cura a Carattere Scientifico) Istituto Neurologico Carlo Besta. Prognostic factors for survival in 676 consecutive patients with newly diagnosed primary glioblastoma. Neuro Oncol. 2008 Feb;10(1):79-87.

17. Weller M, Felsberg J, Hartmann C et al. Molecular predictors of progression-free and overall survival in patients with newly diagnosed glioblastoma: a prospective translational study of the German Glioma Network. J Clin Oncol. 2009 Dec 1;27(34):5743-50.

18. Sonoda Y, Kumabe T, Watanabe M et al. Long-term survivors of glioblastoma: clinical features and molecular analysis. Acta Neurochir. 2009 Nov;151(11):1349-58.

19. Kreth FW, Thon N, Simon M et al. German Glioma Network. Gross total but not incomplete resection of glioblastoma prolongs survival in the era of radiochemotherapy. Ann Oncol. 2013 Dec;24(12):3117-23

20. Fekete B, Werlenius K, Örndal C, Rydenhag B. Prognostic factors for glioblastoma patients-a clinical population-based study. Acta Neurol Scand. 2016 Jun;133(6):434-41.

21. Nakagawa Y, Sasaki H, Ohara K et al. Clinical and Molecular Prognostic Factors for Long-Term Survival of Patients with Glioblastomas in Single-Institutional Consecutive Cohort. World Neurosurg. 2017 Oct; 106:165-173.

22. Glenn CA, Baker CM, Conner AK et al. An Examination of the Role of Supramaximal Resection of Temporal Lobe Glioblastoma Multiforme. World Neurosurg. 2018 Jun;114:e747-e755.

23. Roh TH, Kang SG, Moon JH et al. Survival benefit of lobectomy over gross-total resection without lobectomy in cases of glioblastoma in the non-eloquent area: a retrospective study. J Neurosurg. 2019 Mar 1:1-7.

24. Molinaro AM, Hervey-Jumper S, Morshed RA et al. Association of Maximal Extent of Resection of Contrast-Enhanced and Non-Contrast-Enhanced Tumor With Survival Within Molecular Subgroups of Patients With Newly Diagnosed Glioblastoma. JAMA Oncol. 2020 Apr 1;6(4):495-503.

25. Stupp R, Taillibert S, Kanner A et al. Effect of Tumor-Treating Fields Plus Maintenance Temozolomide vs Maintenance Temozolomide Alone on Survival in Patients with Glioblastoma: A Randomized Clinical Trial. JAMA. 2017 Dec 19;318(23):2306-2316.

26. Krex D, Klink B, Hartmann C et al. German Glioma Network. Long-term survival with glioblastoma multiforme. Brain 2007 Oct;130(Pt 10):2596-606.

27. Scott JN, Rewcastle NB, Brasher PM et al. Which glioblastoma multiforme patient will become a long-term survivor? A population-based study. Ann Neurol. 1999 Aug;46(2):183-8.

28. McLendon RE, Halperin EC. Is the long-term survival of patients with intracranial glioblastoma multiforme overstated? Cancer. 2003 Oct 15;98(8):1745-8.

29. Darefsky AS, King JT Jr, Dubrow R. Adult glioblastoma multiforme survival in the temozolomide era: a population-based analysis of Surveillance, Epidemiology, and End Results registries. Cancer. 2012 Apr 15;118(8):2163-72.

30. Garcia CR, Slone SA, Dolecek TA, Huang B, Neltner JH, Villano JL. Primary central nervous system tumor treatment and survival in the United States, 2004-2015. J Neurooncol. 2019 Aug;144(1):179-191.

31. Brodbelt A, Greenberg D, Winters T, Williams M, Vernon S, Collins VP; (UK) National Cancer Information Network Brain Tumour Group. Glioblastoma in England: 2007-2011. Eur J Cancer. 2015 Mar;51(4):533-42.

32. Jung KW, Yoo H, Kong HJ, Won YJ, Park S, Lee SH. Population-based survival data for brain tumors in Korea. J Neurooncol. 2012 Sep;109(2):301-7.

33. Visser O, Ardanaz E, Botta L, Sant M, Tavilla A, Minicozzi P; EUROCARE-5 Working Group. Survival of adults with primary malignant brain tumours in Europe; Results of the EUROCARE-5 study. Eur J Cancer. 2015 Oct;51(15):2231-2241

34. Narita Y, Shibui S. Committee of Brain Tumor Registry of Japan Supported by the Japan Neurosurgical Society. Trends and Outcomes in the Treatment of Gliomas Based on Data during 2001-2004 from the Brain Tumor Registry of Japan. Neurol Med Chir (Tokyo). 2015;55 Suppl 1:286-95. 
35. Fuentes-Raspall R, Solans M, Roca-Barceló A et al. Descriptive epidemiology of primary malignant and non-malignant central nervous tumors in Spain: Results from the Girona Cancer Registry (1994-2013). Cancer Epidemiol. 2017 Oct;50(Pt A):1-8.

36. Cantrell JN, Waddle MR, Rotman M et al. Progress Toward Long-Term Survivors of Glioblastoma. Mayo Clin Proc. 2019 Jul;94(7):1278-1286.

37. Stupp R, Hegi ME, Mason WP et al. European Organisation for Research and Treatment of Cancer Brain Tumour and Radiation Oncology Groups; National Cancer Institute of Canada Clinical Trials Group. Effects of radiotherapy with concomitant and adjuvant temozolomide versus radiotherapy alone on survival in glioblastoma in a randomised phase III study: 5-year analysis of the EORTC-NCIC trial. Lancet Oncol. 2009 May;10(5):459-66.

38. Kraus JA, Wenghoefer M, Schmidt MC et al. Long-term survival of glioblastoma multiforme: importance of histopathological reevaluation. J Neurol. 2000 Jun;247(6):455-60.

39. Ullén H, Mattsson B, Collins VP. Long-term survival after malignant glioma. A clinical and histopathological study on the accuracy of the diagnosis in a population-based cancer register. Acta Oncol. 1990;29(7):875-8.

40. Amelot A, De Cremoux P, Quillien V et al. IDH-Mutation Is a Weak Predictor of Long-Term Survival in Glioblastoma Patients. PLoS One. 2015 Jul 9;10(7):e0130596.

41. Lu J, Cowperthwaite MC, Burnett MG, Shpak M. Molecular Predictors of Long-Term Survival in Glioblastoma Multiforme Patients. PLoS One. 2016 Apr 28;11(4):e0154313.

42. Cantero D, Rodríguez de Lope Á, Moreno de la Presa R et al. Molecular Study of Long-Term Survivors of Glioblastoma by Gene-Targeted Next-Generation Sequencing. J Neuropathol Exp Neurol. 2018 Aug 1;77(8):710-716.

43. Bowles AP Jr, Perkins E Long-term remission of malignant brain tumors after intracranial infection: a report of four cases. Neurosurgery. 1999 Mar;44(3):636-42; discussion 642-3.

44. Bohman LE, Gallardo J, Hankinson TC et al. The survival impact of postoperative infection in patients with glioblastoma multiforme. Neurosurgery. 2009 May;64(5):828-34; discussion 834-5.

45. Chen YR, Ugiliweneza B, Burton E, Woo SY, Boakye M, Skirboll S. The effect of postoperative infection on survival in patients with glioblastoma. J Neurosurg. 2017 Oct;127(4):807-811.

46. Cahoon EK, Inskip PD, Gridley G, Brenner AV. Immune-related conditions and subsequent risk of brain cancer in a cohort of 4.5 million male US veterans. Br J Cancer. 2014 Apr 2;110(7):1825-33.

47. Hemminki K, Liu X, Försti A, Ji J, Sundquist J, Sundquist K. Subsequent brain tumors in patients with autoimmune disease. Neuro Oncol. 2013 Sep;15(9):1142-50.

48. Mattson B, Wallgren A. Completeness of the Swedish Cancer Register. Non-notified cancer cases on death certificates in 1978. Acta Radiol Oncol 1984; 23:305-13.

49. Barlow L, Westergren K, Holmberg L, Talbäck M. The completeness of the Swedish Cancer Register: a sample survey for year 1998. Acta Oncol. 2009;48(1):27-33.

50. Tettamanti G, Ljung R, Ahlbom A et al. Central nervous system tumor registration in the Swedish Cancer Register and Inpatient Register between 1990 and 2014. Clin Epidemiol. 2019 Jan 4; 11:81-92.

51. Kleihues P, Burger PC, Scheithauer BW. The new WHO classification of brain tumours. Brain Pathol. 1993 Jul;3(3):25568.

52. Tykocki T, Eltayeb M. Ten-year survival in glioblastoma. A systematic review. J Clin Neurosci. 2018 Aug;54:7-13.

53. Marko NF, Weil RJ, Schroeder JL, Lang FF, Suki D, Sawaya RE. Extent of resection of glioblastoma revisited: personalized survival modeling facilitates more accurate survival prediction and supports a maximum-safe-resection approach to surgery. J Clin Oncol. 2014 Mar 10;32(8):774-82.

54. Posti JP, Bori M, Kauko T et al. Presenting symptoms of glioma in adults. Acta Neurol Scand. 2015 Feb;131(2):88-93.

55. Lehrer S, Rheinstein PH, Rosenzweig KE. Allergy may confer better survival on patients with gliomas. Clin Neurol Neurosurg. 2019 Feb; 177:63-67

Page 10/19 
56. Schlehofer B, Blettner M, Preston-Martin S et al. Role of medical history in brain tumour development. Results from the international adult brain tumour study. Int J Cancer. 1999 Jul 19;82(2):155-60.

57. Schwartzbaum J, Jonsson F, Ahlbom A et al. Cohort studies of association between self-reported allergic conditions, immune-related diagnoses and glioma and meningioma risk. Int J Cancer. 2003 Sep 1;106(3):423-8.

58. Wiemels JL, Wiencke JK, Patoka J et al. Reduced immunoglobulin E and allergy among adults with glioma compared with controls. Cancer Res. 2004 Nov 15;64(22):8468-73.

59. Linos E, Raine T, Alonso A, Michaud D. Atopy and risk of brain tumors: a meta-analysis. J Natl Cancer Inst. 2007 Oct 17;99(20):1544-50.

60. Wigertz A, Lönn S, Schwartzbaum J et al. Allergic conditions and brain tumor risk. Am J Epidemiol. 2007 Oct 15;166(8):941-50.

61. Costanza M, Finocchiaro G. Allergic Signs in Glioma Pathology: Current Knowledge and Future Perspectives. Cancers (Basel). 2019 Mar 22;11(3).

62. Zacharia BE, DiStefano N, Mader MM et al. Prior malignancies in patients harboring glioblastoma: an institutional casestudy of 2164 patients. J Neurooncol. 2017 Sep;134(2):245-251.

63. De Bonis $\mathrm{P}$, Albanese A, Lofrese $\mathrm{G}$ et al. Postoperative infection may influence survival in patients with glioblastoma: simply a myth? Neurosurgery. 2011 Oct;69(4):864-8; discussion 868-9.

64. Mineo JF, Bordron A, Baroncini M et al. Prognosis factors of survival time in patients with glioblastoma multiforme: a multivariate analysis of 340 patients. Acta Neurochir (Wien). 2007 Mar;149(3):245-52; discussion 252-3.

65. Paravati AJ, Heron DE, Landsittel D et al. Radiotherapy and temozolomide for newly diagnosed glioblastoma and anaplastic astrocytoma: validation of Radiation Therapy Oncology Group-Recursive Partitioning Analysis in the IMRT and temozolomide era. J Neurooncol. 2011 Aug;104(1):339-49.

66. Thumma SR, Fairbanks RK, Lamoreaux WT et al. Effect of pretreatment clinical factors on overall survival in glioblastoma multiforme: a Surveillance Epidemiology and End Results (SEER) population analysis. World J Surg Oncol. 2012 May 3;10:75.

67. Chaudhry NS, Shah AH, Ferraro $\mathrm{N}$ et al. Predictors of long-term survival in patients with glioblastoma multiforme: advancements from the last quarter century. Cancer Invest. 2013 Jun;31(5):287-308.

68. Adeberg S, Bostel T, König L, Welzel T, Debus J, Combs SE. A comparison of long-term survivors and short-term survivors with glioblastoma, subventricular zone involvement: a predictive factor for survival? Radiat Oncol. 2014 Apr 23;9:95.

69. Albert FK, Forsting M, Sartor K, Adams HP, Kunze S. Early postoperative magnetic resonance imaging after resection of malignant glioma: objective evaluation of residual tumor and its influence on regrowth and prognosis. Neurosurgery. 1994 Jan;34(1):45-60; discussion 60-1.

70. Lacroix M, Abi-Said D, Fourney DR et al. A multivariate analysis of 416 patients with glioblastoma multiforme: prognosis, extent of resection, and survival. J Neurosurg. 2001 Aug;95(2):190-8.

71. Sanai N, Polley MY, McDermott MW, Parsa AT, Berger MS. An extent of resection threshold for newly diagnosed glioblastomas. J Neurosurg. 2011 Jul;115(1):3-8.

72. Grabowski MM, Recinos PF, Nowacki AS et al. Residual tumor volume versus extent of resection: predictors of survival after surgery for glioblastoma. J Neurosurg. 2014 Nov;121(5):1115-23.

73. Almeida JP, Chaichana KL, Rincon-Torroella J, Quinones-Hinojosa A. The value of extent of resection of glioblastomas: clinical evidence and current approach. Curr Neurol Neurosci Rep. 2015;15(2):517.

74. Johnson DR, Ma DJ, Buckner JC, Hammack JE. Conditional probability of long-term survival in glioblastoma: a population-based analysis. Cancer. 2012 Nov 15;118(22):5608-13.

75. Poon MTC, Sudlow CLM, Figueroa JD, Brennan PM. Longer-term ( $\geq 2$ years) survival in patients with glioblastoma in population-based studies pre- and post-2005: a systematic review and meta-analysis. Sci Rep. 2020; 10: 11622. 
76. Martinez R, Schackert G, Yaya-Tur R, Rojas-Marcos I, Herman JG, Esteller M. Frequent hypermethylation of the DNA repair gene MGMT in long-term survivors of glioblastoma multiforme. J Neurooncol. 2007 May;83(1):91-3.

77. Reifenberger G, Weber RG, Riehmer V et al. German Glioma Network. Molecular characterization of long-term survivors of glioblastoma using genome- and transcriptome-wide profiling. Int J Cancer. 2014 Oct 15;135(8):1822-31.

78. Gerber NK, Goenka A, Turcan S et al. Transcriptional diversity of long-term glioblastoma survivors. Neuro Oncol. 2014 Sep;16(9):1186-95.

79. Burgenske DM, Yang J, Decker PA et al. Molecular Profiling of Long-Term IDH-wildtype Glioblastoma Survivors. Neuro Oncol. 2019 Nov 4;21(11):1458-1469

80. Pekmezci M, Rice T, Molinaro AM et al. Adult infiltrating gliomas with WHO 2016 integrated diagnosis: additional prognostic roles of ATRX and TERT. Acta Neuropathol. 2017 Jun;133(6):1001-1016

81. Gomes I, Moreno DA, Dos Reis MB et al. Low MGMT digital expression is associated with a better outcome of IDH1 wildtype glioblastomas treated with temozolomide. J Neurooncol. 2021 Jan;151(2):135-144

\section{Tables}

Table 1. Distribution of total HGG cases, HGG 5-year survivors and verified 5-year GBM survivors

\begin{tabular}{lccr}
\hline Time Cohorts & Total HGG & HGG 5YS, No (\%) & GBM 5YS, No (\%) \\
\hline $1958-1969$ & 3291 & $188(5.7)$ & $10(0.4)$ \\
$1970-1979$ & 3119 & $149(4.7)$ & $19(0.6)$ \\
$1980-1989$ & 3193 & $187(5.8)$ & $21(0.7)$ \\
$1990-1999$ & 3162 & $212(6.7)$ & $27(0.9)$ \\
\hline $1958-1999$ & 12765 & $736(5.8)$ & $77(0.6)$ \\
\hline
\end{tabular}

HGG = High-Grade Glioma

$\mathrm{GBM}=$ Glioblastoma

$5 \mathrm{YS}=$ Five-year survivors

Table 2. The histopathological diagnosis in 585 five-year survivors with HGG according to the ICD 7, ICD 9, ICD 0 and ICD 10 classifications. 


\begin{tabular}{|c|c|c|c|}
\hline Diagnosis & & No & $\%$ \\
\hline Anaplastic Astrocytoma gr III & & 197 & 33.7 \\
\hline Astrocytoma gr II & & 86 & 14.7 \\
\hline Glioblastoma & & 77 & 13.2 \\
\hline Oligoastrocytoma gr III & & 34 & 5.8 \\
\hline Pilocytic astrocytoma gr I & 27 & & 4.6 \\
\hline Oligodendroglioma gr II & 26 & & 4.4 \\
\hline Anaplastic oligodendroglioma gr III & 24 & & 4.1 \\
\hline Spongioblastoma (cerebellum) & 21 & & 3.6 \\
\hline Oligoastrocytoma gr II & 19 & & 3.2 \\
\hline Meningioma & 19 & & 3.2 \\
\hline No detectable PAD & 18 & & 3.1 \\
\hline Malignant glioma NOS & 13 & & 2.2 \\
\hline Ganglioglioma gr I & 5 & & 0.9 \\
\hline Ependymoma gr III & 4 & & 0.7 \\
\hline Ependymoma gr II & 4 & & 0.7 \\
\hline Intramedullary glioma tumor & 3 & & 0.5 \\
\hline Ganglioglioma gr III & 2 & & 0.3 \\
\hline DNET & 2 & & 0.3 \\
\hline Ganglioglioma gr II & 1 & & 0.2 \\
\hline Hemangioblastoma gr 1 & 1 & & 0.2 \\
\hline Ependymoma grade I & 1 & & 0.2 \\
\hline
\end{tabular}

HGG = High Grade Glioma

Table 3. Characteristics of GBM five-year survivors from retrieved medical charts, $\mathrm{N}=73$. 


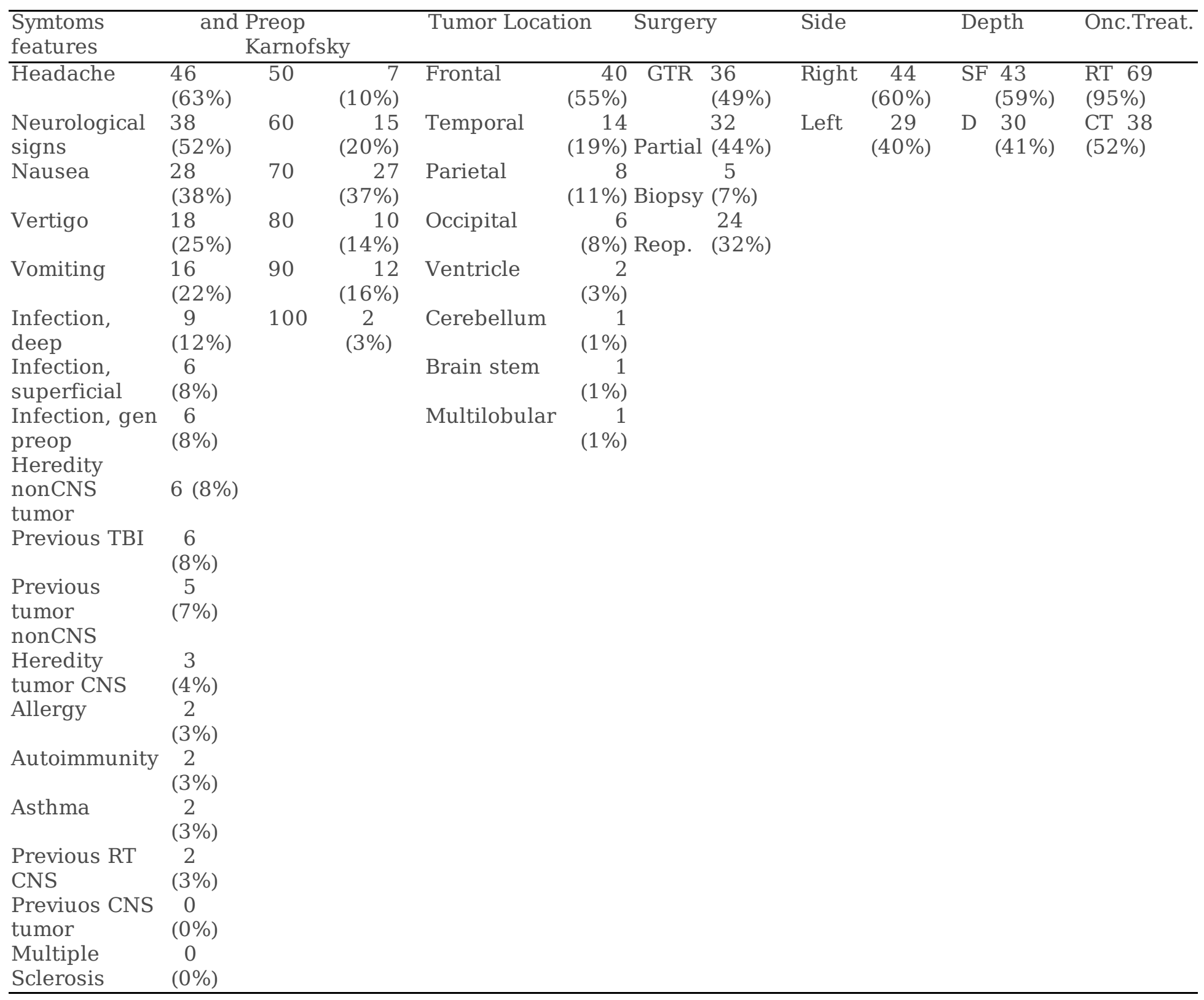

GTR= Gros Total Resection; SF = superficial; D = Deep; RT = radiotherapy; CT = chemotherapy; Onc. Treat; = oncological treatment; GBM = Glioblastoma

\section{Figures}


High-Grade Glioma, diagnosed 1958-1999 in the

Swedish Cancer Registry

$$
\mathrm{N}=12765
$$

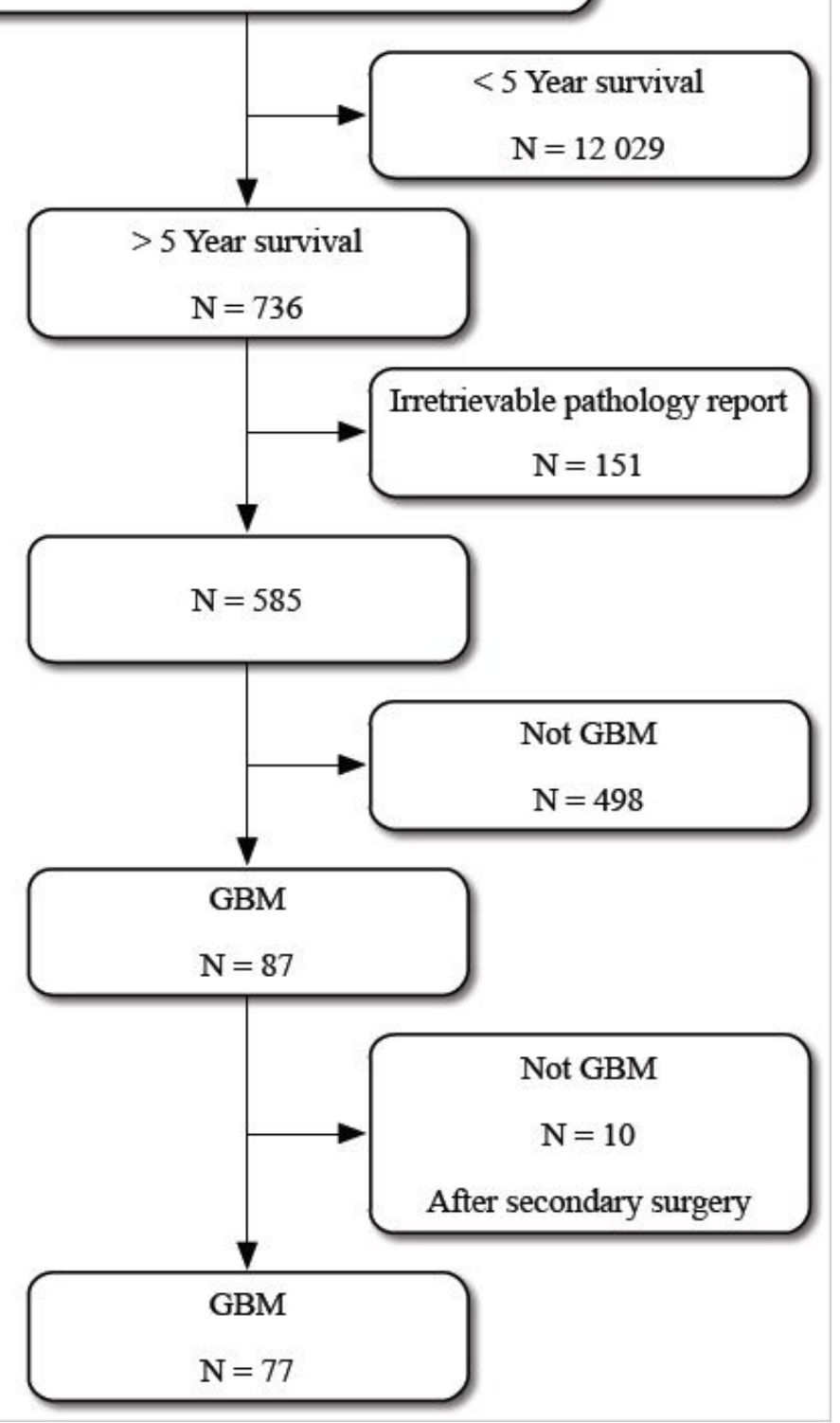

\section{Figure 1}

Flow chart of high-grade glioma and glioblastoma five-year survivors from the Swedish Cancer Registry and the Swedish Cause of Death Register between 1958-1999. 


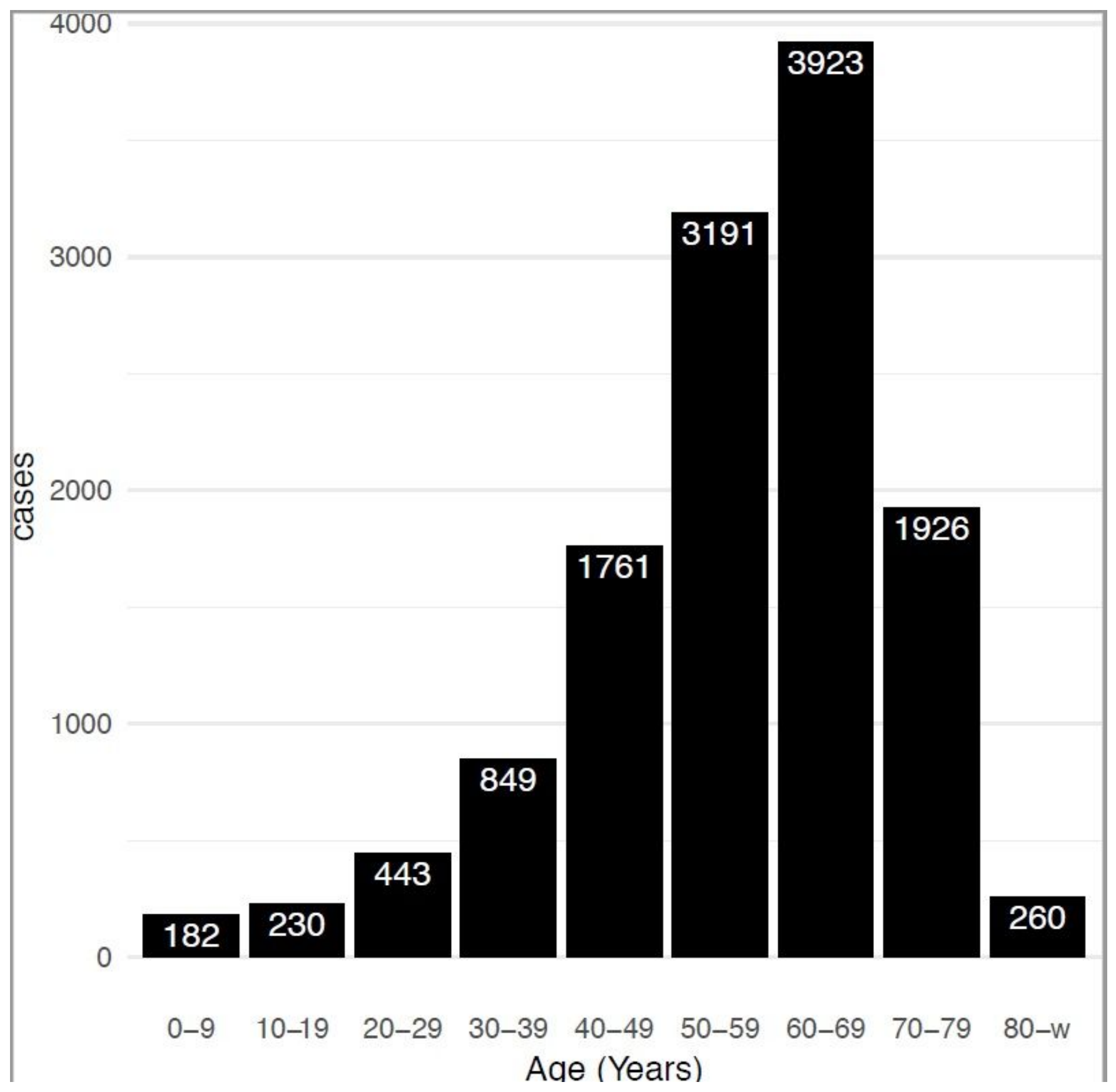

Figure 2

Age distribution of patients with number of patients added to each bar. 

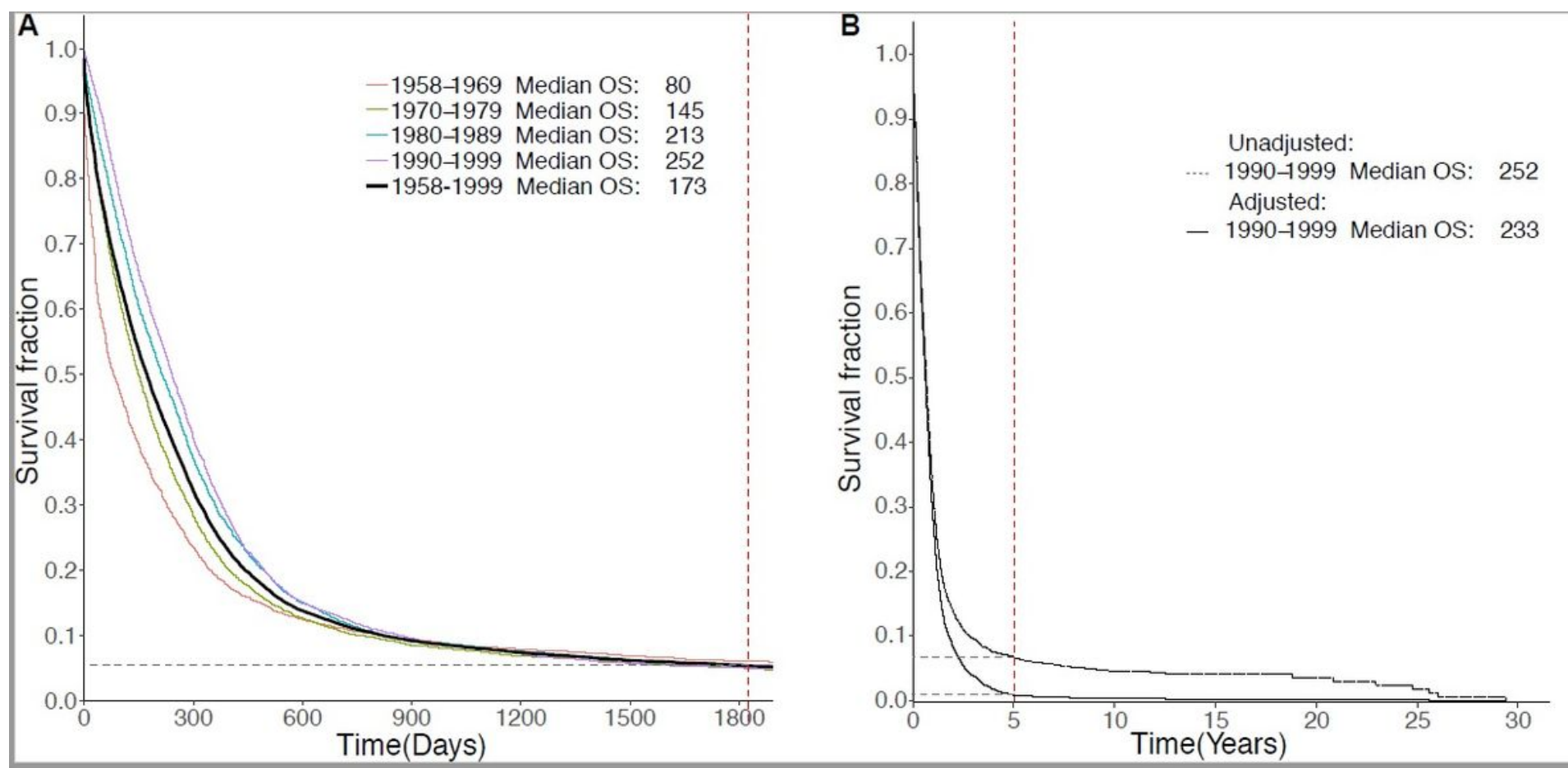

Figure 3

a. Patient survival. Patients divided in four time-period cohorts. Median survival is noted. b. Patient survival. Adjusted 90-99 time cohort when all pathology reports were retrieved. Median survival is noted. 


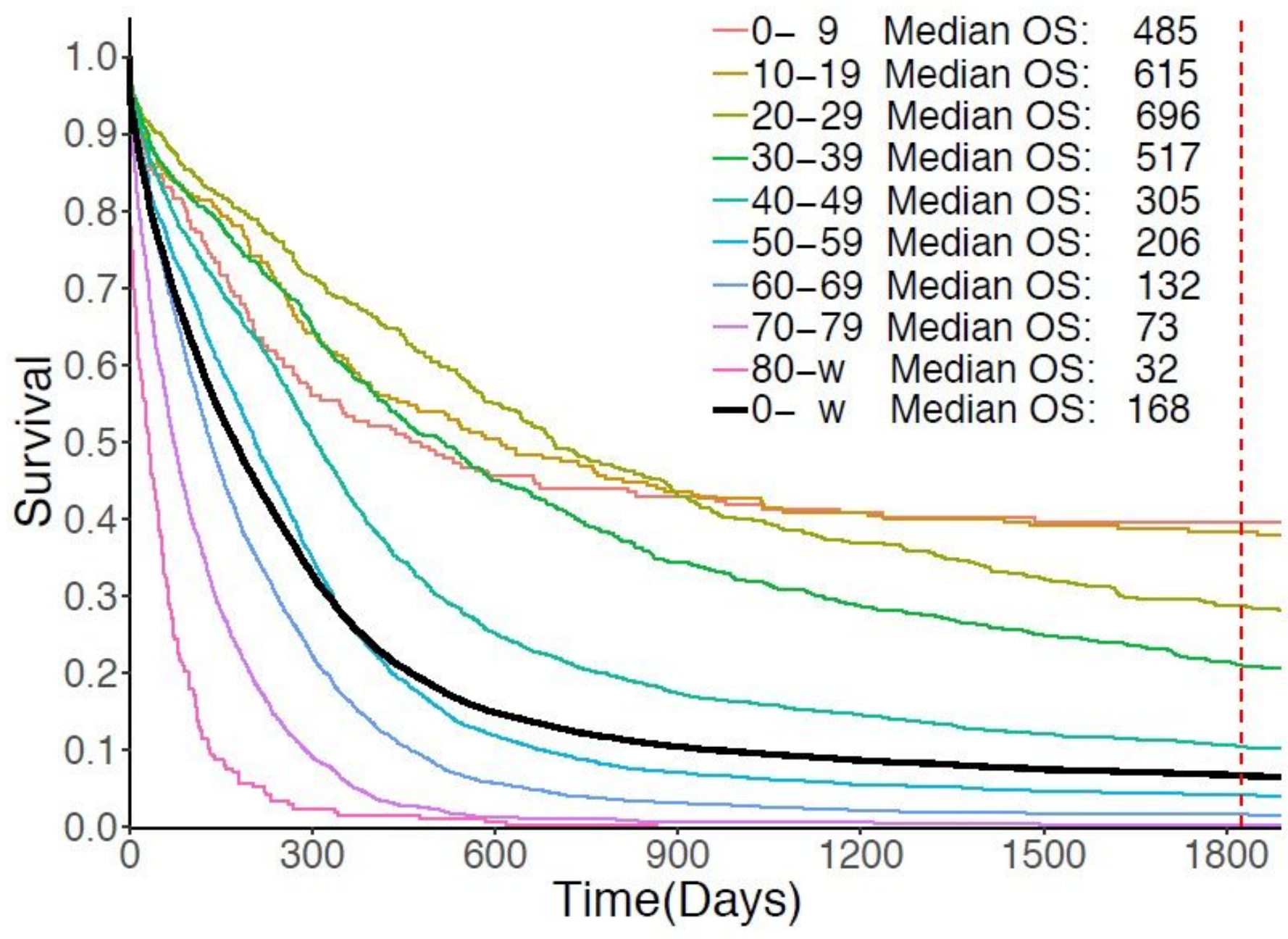

Figure 4

Patient survival. Patients divided into age-groups. Median survival is noted.

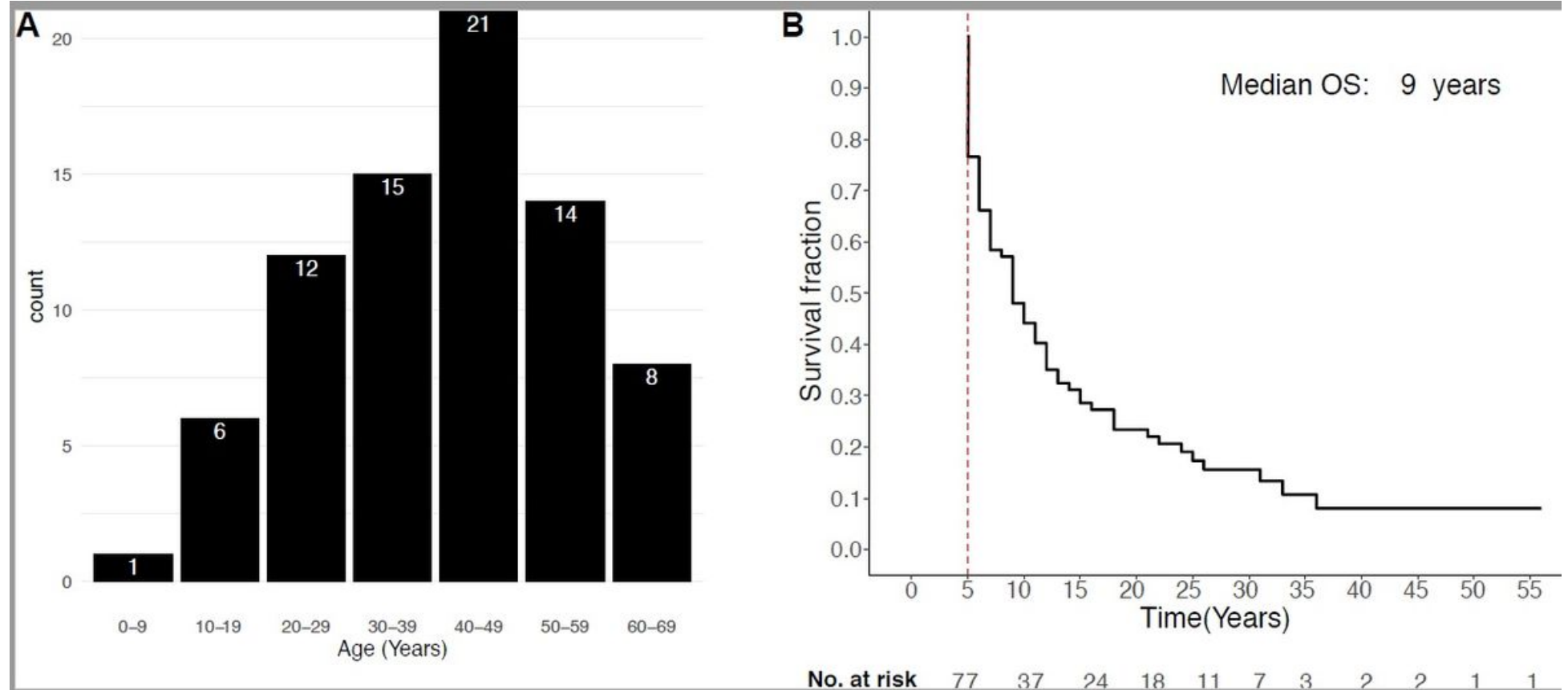




\section{Figure 5}

Age distribution of glioblastoma five-year survivors when diagnosed. Number of patients added to each bar. b. Overall survival of glioblastoma five-year survivors with median OS noted. 